\title{
Moralische Leistung und Auswirkungen von Gefangenschaft
}

\section{Zusammenfassung}

Noch nie hat mehr für eine sorgfältige Untersuchung der Erfahrung der Gefangenschaft und ihrer Wirkungen gesprochen. Richard Lippke und Lö̈c Wacquant haben erst kürzlich wichtige Untersuchungen zur ungerechten Anwendung des Freiheitsentzugs sowie der Ausweitung der strafrechtlichen Regelung von Problemen mit Not und Armut in Europa und den USA durchgeführt. Neben den Problemen des ausufernden Gebrauchs von Freiheitsentzug und seiner ungleichen Vollstreckung sind in Strafanstalten auch ungleichmäßige Bestrafung und Leid zu beobachten. Eine durch die Autorin durchgeführte Studie über zwölf kommunale Männer- und Frauenstrafvollzugsanstalten hat ergeben, dass eine der Einrichtungen deutlich besser zu „überleben“ war als die anderen. Bemerkenswert ist, dass es sich dabei um eine privat betriebene Institution handelt. Der Grad der durch die Gefangenen erlebten Belastungen war insgesamt extrem hoch. Jedoch variierten diese Belastungen zwischen den Anstalten in einer Weise, die nicht allein durch individuelle Eigenschaften erklärt werden kann. Diese Unterschiede standen in einem systematischen Zusammenhang zu Suizidraten und zu wichtigen Aspekten des Vollzugsregimes wie Sicherheit, Fairness, Kultur und Beziehungen zwischen Bediensteten und Gefangenen. Diese empirischen Fakten sagen eine Menge über das Wesen der Erfahrung von Gefangenschaft, über die Unterschiede zwischen verschiedenen Strafvollzugsanstalten und die Unzulänglichkeit unserer Annahmen über eine gerechte und angemessene Strafvollstreckung aus. Wir sollten über die Darstellung der Ausweitung des Freiheitsentzugs hinaus unser wissenschaftliches Augenmerk auch darauf richten, „wie sich die Bestrafung anfühlt“, wie der Strafvollzug sich mit der Zeit verändert und welche Zusammenhänge zwischen diesen Fakten und Auswirkungen auf die Gefangenen bestehen.

My whole heart and soul ache with the pain of being here (Boyle 1985: 71).

Stress can contaminate programmes, undermine adjustment efforts, and leave a residue of bitterness and resentment among inmates. It can make the prison a destructive and debi- litating institution (Johnson and Toch 1982: 20).

\section{Einführung}

Diesen Beitrag schreibe ich als Verfechterin der Erforschung der Auswirkungen von Freiheitsentzug. Dieses Thema ist sowohl methodisch als auch konzeptionell eine Herausforderung. Unsere gesammelten empirischen Befunde sind von akuter theoretischer, sozialer und politischer Bedeutung. Die „Erfahrung der Gefangenschaft“ durchläuft wichtige Veränderungen im Zuge der anhaltenden Ausweitung und Modernisierung von Haftanstalten, die trotz einiger Fortschritte im Bereich des Menschenrechtsschutzes ihren Zugriff auf ihre Bewohner verstärken. Die offiziell eingeräumten Zwekke, Entbehrungen, Schäden und weitergehenden Wirkungen des Strafvollzugs ändern sich mit der Wandlung der strafrechtlichen Befindlichkeiten und der Organisation des Strafvollzugs. In Großbritannien werden zurzeit Kernziele des Strafvollzugs verschoben: weg von Rehabilitation in Richtung einer verstärkten Betonung des Schutzes der Öffentlichkeit. ${ }^{1}$ Welche Auswirkungen mag diese Verschiebung auf die Politik, die Praxis und die Ergebnisse haben? Es ist eine Herausforderung, in diesem Bereich empirisch sorgfältig und konzeptionell klar zu arbeiten, den Zugang zum Forschungsfeld überhaupt erst zu schaffen und auch aufrechtzuerhalten und zu wissen, wie man Forschungsergebnisse nützlich und zugleich verantwortungsvoll anwendet und auf ihnen aufbaut. Dies ist unsere gemeinsame Verantwortung als Strafvollzugsforscherinnen und -forscher.

In seinem Buch Rethinking Imprisonment (wörtlich übersetzt: „Den Strafvollzug überdenken“ - d. Übers.) hat Richard Lippke erst kürzlich argumentiert, dass ein großer Teil der zeitgenössischen Anwendung des Freiheitsentzugs als strafrechtliche Sanktion - sein Wesen, sein Nutzen und seine Dauer - nicht gerechtfertigt ist. Lippke schlägt vor, Vollzugsbedingungen zu schaffen, die an der unteren Grenze heutiger Mindeststandards des Strafvollzugs oder knapp darüber liegen und die Bewegungs- und Versammlungsfreiheit sowie die Privatsphäre von Gefangenen erheblich einschränken. Zugleich sollen diese Bedingungen das geistige und körperliche Wohlbefinden der Gefangenen, Arbeit und Familienleben, sowie die Wahrnehmung bürgerlicher und politischer Rechte gewährleisten. Während des Freiheitsentzugs sollte die Einstellung eines Gefangenen zu seiner Rolle als verantwortungsvoller Bürger weiterentwickelt und nicht untergraben werden; seine Fähigkeit zu moralischen Überlegungen und Entscheidungen sollte verbessert und nicht beschädigt werden. Vollzugsformen, die diese Bedingungen nicht einhalten, stoßen an eine moralische Grenze (Lippke 2007). Auch Lö̈c Wacquant lieferte eine wichtige Untersuchung zur "Ausdehnung des Strafvollzugselends" durch harsche, vergeltende Kriminalpolitik in Europa und den USA. Wacquant stellt die These auf, dass der Einsatz des Gefängnisses illegitim und untrennbar mit Armut und dem Arbeitsmarkt verbunden sei (Wacquant 1999; s. auch Deflau 2001).

Theoretische, rechtliche und vielleicht insbesondere politische Auseinandersetzungen über den Nutzen von Freiheitsentzug sind immer komplexer und zugleich widersprüchlich geworden. In noch nicht allzu ferner Vergangenheit wurde geschrieben, dass der Freiheitsentzug kein „unnötiges und mutwilliges Leid“ zufügen darf (z. B. Dunn, in Johnson/Toch 1982: 11). Die Rechtsprechung hat deutlich formuliert, dass Gefangene lediglich ihre Freiheit verlieren und keine anderen Rechte, sofern sie nicht ausdrücklich durch die Gefangenschaft entzogen werden (Lord Wilberforce in Raymond v Honey 1982). ${ }^{2}$ Diese gängige Wahrnehmung der Wirkung und der Zielsetzungen des Freiheitsentzugs wird inzwischen jedoch angezweifelt, so dass manche der als selbstverständlich angesehenen Grenzen bei der Frage, wie viel Gefangenschaft berechtigt ist oder wie schmerzlich das Leben in Haft sein sollte, zurzeit auf die Probe gestellt werden. Während des letzten Jahrhunderts herrschte zumindest offiziell ein rechtlicher und politischer Konsens, der am besten von Sir Alexander Paterson ausgedrückt wurde, der sagte, dass Gefängnisse „should be used as punishment and not for punishment" (Ruck 1951: 23), also dass der Freiheitsentzug die Strafe sei und nicht die Gelegenheit zur Be- 
strafung. Winston Churchill sagte zu diesem Thema, dass eine Freiheitsstrafe einen Mann in einen anständigen Bürger verwandeln solle. ${ }^{3}$ Der Britische Justizminister Jack Straw brach in einer umstrittenen Weise mit diesem Konsens in einem Vortrag vor der Royal Society of Arts 2008, in dem er sagte, dass Gefängnisse "are for punishment and reform", also zur Bestrafung und Veränderung da seien (Straw 2008). Wir sind uns in sozialer Hinsicht meiner Meinung nach weniger sicher, was rechtschaffen und tugendhaft oder was gerecht ist und sind auch weniger dem Menschenfreundlichen verpflichtet - eine Meinung, die andere teilen. ${ }^{4}$ Argumente für eine Mäßigung und Zurückhaltung in der Strafjustiz (s. z. B. Loader 2008) erfordern eine sorgfältige Darstellung und empirische Belege. Die Ergebnisse sind in jeder Hinsicht dürftig. Aber die Erforschung wichtiger Unterschiede bringt uns voran.

Suizid im Gefängnis ist ein Thema, mit dem ich mich im Laufe meiner Karriere in der Strafvollzugsforschung wiederholt befasst habe. Hier bietet sich die Untersuchung von Fragen nach den relativen Einflüssen individueller Eigenschaften und solchen der Umwelt auf die Erklärung von zwei extremen Folgen der Gefangenschaft an: Überleben oder Verzweiflung. Einige Forschungsprojekte dazu haben mich bewegt, intensiv - aber dennoch unzureichend - darüber nachzudenken, was die Begriffe Leid, Belastung und Wohlbefinden im Kontext des Strafvollzugs bedeuten. Ein großer Teil der frühen Forschung beruhte auf engen und oberflächlichen oder irreführenden operationalen Definitionen dieser Begriffe (z. B. eine Annahme, dass Leid ein im Zeitverlauf messbarer intellektueller Verfall sei). Außerdem wurde die Forschung durch die irrige Trennung psychologischer und soziologischer Methoden und Perspektiven behindert. Cohen und Taylors Meilenstein Psychological Survival (1972; wörtlich übersetzt: Psychologisches Überleben), Zimbardos berüchtigte Haftsimulation (2007), und Sykes (1958) klassische Beschreibung von Haftdeprivationen liefern allesamt plausible konzeptionelle Darstellungen der Auswirkungen des Freiheitsentzug. Jedoch wurden diese Studien weithin wegen ihrer wenig durchdachten oder mangelhaften Methodik kritisiert (Walker 1987).

Der aktuelle Stand der Dinge ist in vieler Hinsicht eine erhebliche Verbesserung. Eine neue Generation von Sozialforschern kombiniert enthusiastisch psychologische Methoden mit soziologischen Konzepten.
Die Mess- und Analysetechniken verbessern sich fortwährend. Die Technik ist auf unserer Seite, und der Zugang zu Strafanstalten ist insgesamt in größerem Umfang möglich, als es früher der Fall war. Private und staatliche Geldgeber stellen Forschungsmittel zur Verfügung. Jedoch ist unser Verständnis der Auswirkungen von Gefangenschaft immer noch sehr begrenzt. Forschungsergebnisse werden vielleicht nicht öffentlich wahrgenommen. Sie werden von Missverständnissen und Erwartungen der Öffentlichkeit verdrängt. Gefängnisse und das, was hinter den Mauern abläuft, haben sich verändert. Einen Beitrag zu leisten zu einer aufgeklärten öffentlichen und politischen Debatte über die Nutzen und Wirkungen des Strafvollzugs wird eine erhebliche, koordinierte Anstrengung und Strategie erfordern. Ich hoffe, dass einige neue Zentren der Strafvollzugsforschung zur Erreichung dieses Ziels beitragen werden.

In diesem Beitrag stelle ich die These auf, dass sowohl bezüglich der psychologischen Bedingungen als auch der sozialen und theoretischen Zwecke des Strafvollzugs viele Missverständnisse herrschen, und dass Strafvollzugsbehörden aufgrund dieses Mangels an Verständnis die grundlegenden Standards der Fairness verletzen. Ergebnisse empirischer Forschung stellen viele unserer Annahmen über den angemessenen und gerechten Gebrauch von Freiheitsentzug in Frage. In England und Wales sind Übertretungen aller von Lippke aufgeführten Anforderung an den Strafvollzug häufig und weit verbreitet. Eine sorgfältige Untersuchung, inwieweit sie von Strafanstalten gebrochen oder eingehalten werden, differenziert nach Gerichtsbezirk oder privatem oder staatlichem Betreiber, zeigt uns wichtige neue Wege. Es bestünde ein gefährliches Paradox, z. B. falls private Strafanstalten weniger tiefgreifende oder psychologisch belastendere Haftbedingungen schaffen als der Staat, um auf dem neuen „Bestrafungsmarkt“ zu bestehen.

\section{Untersuchungen moralischer Leistung des Strafvollzug}

Ich werde nun vor allem auf vier Studien eingehen, die vier Stationen einer Art noch andauernder Forschungsreise widerspiegeln. Ich werde kurz einige wesentliche Ergebnisse dieser Arbeiten umreißen, von denen drei abgeschlossen sind, und eine in Zusammenarbeit mit den Kollegen Ben Crewe, Clare McLean und Susie Hulley des Cambridge Institute of Criminology noch läuft.
Die erste Untersuchung war ein Versuch, wichtige Dimensionen der Gefängnisqualität, wie sie von Häftlingen und Bediensteten beschrieben wurden, zu identifizieren und zu beurteilen. Dies war ein langer Prozess, der von Abwägung und Beratung geprägt war und mit der Methode der appreciative inquiry an den positiven Erfahrungen der Beteiligten ansetzte. Es kristallisierte sich eine überraschende Übereinstimmung darüber heraus, was „im Strafvollzug zählt“. Die Gefangenen machten deutlich, dass das, was eine Anstalt von anderen unterscheidet, die Art ist, wie sie von den Bediensteten behandelt werden, wie sicher sie sich in der Anstalt fühlen, ob sie ihre Familien treffen können und wie Vertrauen und Macht die Anstalt durchströmen. Wenn sie gefragt wurden, was sie messen würden und sie gebeten und ermuntert wurden, sich damit kreativ und mit einer positiven Herangehensweise auseinanderzusetzen, wurden folgende Faktoren genannt: Respekt, Vertrauen, Beziehungen zwischen Bediensteten und Gefangenen, Sicherheit, Ordnung, Fairness, persönliche Entwicklung, familiärer Kontakt und ihr subjektives Wohlbefinden. Mit ihrer Hilfe haben wir gelernt, genau dies zu tun, und die Ergebnisse sind sehr erhellend. Warum sind diese Merkmale von so großer Bedeutung? Weil es in der menschlichen Erfahrung nichts Schlimmeres gibt, als dass einem die Menschenwürde aberkannt wird und es nichts Wichtigeres gibt als ihre Anerkennung. Wenn Gefangene sagten, „die Bediensteten sollten vorsichtig sein, wenn sie über meine 14jährige Strafe Witze machen", so meinten sie, dass es ihnen - trotz ihrer in dieser Hinsicht geringen Ansprüche - als Mensch etwas ausmacht und sie ändert, wie die Bediensteten mit ihnen über ihre 14jährige Strafe reden. Dies ist wichtiger, als wann sie nachts eingeschlossen werden, steht aber, wie ich an anderer Stelle ausführen werde, auch damit in Zusammenhang (s. Liebling, mit Unterstützung von Arnold, 2004). Die meisten unserer Erfahrungen mit Ereignissen und Institutionen sind im Wesentlichen charakterisiert durch Beziehungen.

Diese Gespräche fanden 2001 in fünf Strafanstalten statt und bildeten unter anderem die Grundlage für einen detaillierteren Fragebogen zu all diesen Belangen des Vollzugserlebnisses. In Bezug auf diese Arbeit spreche ich von moralischer Leistung (moral performance), da die hohe Bewertung (beispielsweise) der Anstaltssicherheit, Fairness und Menschlichkeit durch die Gefangenen bedeutsame Unterschiede zwischen Anstalten ergeben, die ansonsten - nach offiziellen 
Tabelle 1: Anteil der Antworten „stimme zu/ stimme stark zu“ bei Aussagen zum Wohlbefinden

\begin{tabular}{|l|c|c|c|c|c|}
\hline Aussage & Belmarsh & Holme House & Risley & Doncaster & Wandsworth \\
\hline $\begin{array}{l}\text { 1. Die Atmosphäre in dieser Anstalt ist entspannt und } \\
\text { freundlich. }\end{array}$ & $30,1^{\mathrm{b}}$ & 58,5 & 44,5 & $64,0^{\mathrm{a}}$ & 36,6 \\
\hline $\begin{array}{l}\text { 2. Mein Vollzugserlebnis in dieser Anstalt ist stress- } \\
\text { belastet. }\end{array}$ & 57,8 & 36,1 & 48,5 & $26,3^{\mathrm{a}}$ & $61,6^{\mathrm{b}}$ \\
\hline 3. Ich kann in dieser Anstalt ich selbst sein. & $34,9^{\mathrm{b}}$ & 54,3 & 39,7 & $62,3^{\mathrm{a}}$ & 46,6 \\
\hline 4. Ich fühle mich in dieser Anstalt angespannt. & $50,6^{\mathrm{b}}$ & 28,7 & 39,7 & $21,1^{\mathrm{a}}$ & 36,7 \\
\hline 5. Meine Erfahrung in dieser Anstalt ist schmerzhaft. & $50,6^{\mathrm{b}}$ & 25,5 & 31,7 & $15,8^{\mathrm{a}}$ & 35,0 \\
\hline 6. Unter den Gefangenen hier ist die Moral gut. & 24,1 & 41,5 & 20,8 & $48,2^{\mathrm{a}}$ & $16,7^{\mathrm{b}}$ \\
\hline 7. Ich fürchte allgemein um meine psychische Sicherheit. & $28,9^{\mathrm{b}}$ & 21,3 & 24,8 & $15,8^{\mathrm{a}}$ & 28,4 \\
\hline
\end{tabular}

a höchster Wert

$b$ niedrigster Wert

Tabelle 2: Vergleich der Einschätzung des Wohlbefindens durch die Gefangenen: Mittelwerte der einzelnen Items

\begin{tabular}{|l|c|c|c|c|c|c|c|c|c|c|c|c|c|c|c|}
\hline Item & \multicolumn{5}{|c|}{ Score (1-5) } & \multicolumn{6}{|c|}{ ANOVA (Mittelwertdifferenz) } \\
\hline & B & HH & R & D & W & B\&HH & B\&R & B\&D & B\&W & HH\&R & HH\&D & HH\&W & R\&D & R\&W & D\&W \\
\hline $\begin{array}{l}\text { Die Atmosphäre in dieser } \\
\text { Anstalt ist entspannt und } \\
\text { freundlich. }\end{array}$ & $2,89^{\mathrm{b}}$ & 3,34 & 3,08 & $3,55^{\mathrm{a}}$ & 2,95 & 0,45 & $\mathrm{~N} / \mathrm{s}$ & $0,66^{*}$ & $\mathrm{~N} / \mathrm{s}$ & $\mathrm{N} / \mathrm{s}$ & $\mathrm{N} / \mathrm{s}$ & $\mathrm{N} / \mathrm{s}$ & $0,47^{*}$ & $\mathrm{~N} / \mathrm{s}$ & $0,60^{*}$ \\
\hline $\begin{array}{l}\text { Mein Vollzugserlebnis in } \\
\text { dieser Anstalt ist nicht } \\
\text { stressbelastet. }\end{array}$ & $2,34^{\mathrm{b}}$ & 3,06 & 2,55 & $3,29^{\mathrm{a}}$ & 2,42 & $0,73^{*}$ & $\mathrm{~N} / \mathrm{s}$ & $0,95^{* *}$ & $\mathrm{~N} / \mathrm{s}$ & $0,51^{*}$ & $\mathrm{~N} / \mathrm{s}$ & $0,65^{*}$ & $0,74^{* *}$ & $\mathrm{~N} / \mathrm{s}$ & $0,87^{* *}$ \\
\hline $\begin{array}{l}\text { Ich kann in dieser Anstalt } \\
\text { ich selbst sein. }\end{array}$ & $2,82^{\mathrm{b}}$ & 3,20 & 2,94 & $3,47^{\mathrm{a}}$ & 3,08 & $\mathrm{~N} / \mathrm{s}$ & $\mathrm{N} / \mathrm{s}$ & $0,65^{*}$ & $\mathrm{~N} / \mathrm{s}$ & $\mathrm{N} / \mathrm{s}$ & $\mathrm{N} / \mathrm{s}$ & $\mathrm{N} / \mathrm{s}$ & $0,53^{*}$ & $\mathrm{~N} / \mathrm{s}$ & $\mathrm{N} / \mathrm{s}$ \\
\hline $\begin{array}{l}\text { Ich fühle mich in dieser } \\
\text { Anstalt nicht angespannt. }\end{array}$ & $2,54^{\mathrm{b}}$ & 3,21 & 2,95 & $3,46^{\mathrm{a}}$ & 2,98 & $0,67^{*}$ & $\mathrm{~N} / \mathrm{s}$ & $0,92^{* *}$ & $\mathrm{~N} / \mathrm{s}$ & $\mathrm{N} / \mathrm{s}$ & $\mathrm{N} / \mathrm{s}$ & $\mathrm{N} / \mathrm{s}$ & $0,51^{*}$ & $\mathrm{~N} / \mathrm{s}$ & $\mathrm{N} / \mathrm{s}$ \\
\hline $\begin{array}{l}\text { Meine Erfahrung in dieser } \\
\text { Anstalt ist nicht schmerz- } \\
\text { haft. }\end{array}$ & $2,63^{\mathrm{b}}$ & 3,33 & 3,05 & $3,66^{\mathrm{a}}$ & 2,92 & $0,70^{*}$ & $\mathrm{~N} / \mathrm{s}$ & $1,03^{* *}$ & $\mathrm{~N} / \mathrm{s}$ & $\mathrm{N} / \mathrm{s}$ & $\mathrm{N} / \mathrm{s}$ & $\mathrm{N} / \mathrm{s}$ & $0,61^{*}$ & $\mathrm{~N} / \mathrm{s}$ & $0,74^{*}$ \\
\hline $\begin{array}{l}\text { Unter den Gefangenen hier } \\
\text { ist die Moral gut. }\end{array}$ & 2,71 & 3,13 & 2,76 & $3,38^{\mathrm{a}}$ & $2,62^{\mathrm{b}}$ & 0,42 & $\mathrm{~N} / \mathrm{s}$ & $0,67^{* *}$ & $\mathrm{~N} / \mathrm{s}$ & $\mathrm{N} / \mathrm{s}$ & $\mathrm{N} / \mathrm{s}$ & $0,51^{*}$ & $0,61^{* *}$ & $\mathrm{~N} / \mathrm{s}$ & $0,76^{* *}$ \\
\hline $\begin{array}{l}\text { Ich fürchte allgemein nicht } \\
\text { um meine psychische } \\
\text { Sicherheit. }\end{array}$ & $3,14^{\mathrm{b}}$ & 3,50 & 3,33 & $3,68^{\mathrm{a}}$ & 3,27 & $\mathrm{~N} / \mathrm{s}$ & $\mathrm{N} / \mathrm{s}$ & $0,53^{*}$ & $\mathrm{~N} / \mathrm{s}$ & $\mathrm{N} / \mathrm{s}$ & $\mathrm{N} / \mathrm{s}$ & $\mathrm{N} / \mathrm{s}$ & $\mathrm{N} / \mathrm{s}$ & $\mathrm{N} / \mathrm{s}$ & $\mathrm{N} / \mathrm{s}$ \\
\hline
\end{tabular}

a höchster Wert, b niedrigster Wert, * signifikant bei $p<.05$, ** signifikant bei $p<.005, \mathrm{~N} / \mathrm{s}$ : nicht signifikant

B: Belmarsh, HH: Holme House, R: Risley, D: Doncaster, W: Wandsworth

Vergleichskriterien - sehr ähnlich zu sein scheinen (Liebling, mit Unterstützung von Arnold 2004). Ein Ergebnis dieser Studie ist, dass es eine große Diskrepanz zwischen offiziellen Darstellungen des und Bestrebungen für den Strafvollzug und der Gefangenschaft, wie sie die meisten Gefangenen erleben, gibt. Die Gefangenen beschrieben schwankende, aber insgesamt niedrige Grade von Fairness, Respekt, Menschlichkeit, Aktivität und Entlassungsvorbereitung. Sie berichteten von großer Verzweiflung (vor allem in kommunalen Gefängnissen), die bei Strafantritt am größten war.

Tab. 1 zeigt die Unterschiede in den Antworten von 100 zufällig ausgewählten Häft- lingen pro Anstalt zu Fragen zum Anstaltsklima (man beachte z. B. den Unterschied zwischen Belmarsh und Doncaster). Diese Angaben entsprechen weitgehend ihren Einschätzungen anderer Faktoren des Lebens im Strafvollzug. Verwendet wurde eine fünfstufige Likert-Skala. Als besonders belastend wurde der Vollzug in Wandsworth und Belmarsh beschrieben. Die Gefangenen in Doncaster fanden es am ehesten möglich, „man selbst zu sein“ (gefolgt von Holme House), während die Insassen in Belmarsh sich deutlich angespannter fühlten als in den anderen Gefängnissen. ${ }^{5}$

Anhand der Items dieser Dimension kann zwischen allen fünf Anstalten differenziert werden (Tab. 2). Die Ergebnisse zeigen, dass die Gefangenen in Doncaster - dicht gefolgt von Holme House - signifikant höhere Grade des Wohlbefindens aufweisen. Sie berichteten eine signifikant entspanntere Atmosphäre, geringere Belastung durch Stress, mehr Möglichkeiten, „man selbst zu sein“, eine geringere Anspannung, wenig Schmerz, eine bessere Moral unter den Gefangenen und weniger Sorge um ihre „psychische Sicherheit". Belmarsh, am anderen Ende der Skala (und bei manchen Items auch Wandsworth), wurde als deutlich schmerzhafter beschrieben. Die Aussagen 4 und 5 sind hier besonders interessant: $50 \%$ aller Befragten in Belmarsh gaben an, dass sie angespannt 
seien und dass ihre Hafterfahrung schmerzhaft sei. Bei den meisten Items waren auch Unterschiede zwischen Holme House und den übrigen drei Anstalten festzustellen. Die Gefangenen erlebten die Gefangenschaft in der einen Anstalt als schmerzhafter und bestrafender als in der anderen. Man kann aus diesen verschiedenartigen Umsetzungen der Bestrafung wichtige Folgerungen ziehen. Diese Ergebnisse lassen Zweifel an der Angemessenheit der "Proportionalitätsthese “ aufkommen, bei der die Länge einer Freiheitsstrafe der Summe der erlebten Unannehmlichkeit und Bestrafung entspricht (von Hirsch 1976; s. auch von Hirsch/Ashworth 2005).

Tabelle 3: Punktwerte bezüglich „Wohlbefinden"

\begin{tabular}{|l|c|c|}
\hline Anstalt & Reliabilität & Score \\
\hline Doncaster & 0,77 & 3,99 \\
\hline Holme House & 0,80 & 3,25 \\
\hline Risley & 0,88 & 2,95 \\
\hline Wandsworth & 0,82 & 2,89 \\
\hline Belmarsh & 0,79 & 2,72 \\
\hline
\end{tabular}

Die allgemeine interne Reliabilität der Dimension „Wohlbefinden“ für alle Anstalten war mit 0,84 hoch. Zwei Anstalten erzielten Mittelwerte von 3 oder mehr hinsichtlich des Wohlbefindens der Gefangenen (Tab. 3). Es bestehen signifikante Unterschiede zwischen Doncaster und jeweils Holme House, Risley, Wandsworth und Belmarsh sowie zwischen Holme House, Risley und Belmarsh.

Wohlbefinden ist am höchsten und positiv signifikant mit den Dimensionen Fairness (.63), Menschlichkeit (.60) und Macht/Autorität (.60) korreliert. Diese Befunde deuten plausibel darauf hin, dass die Erfahrung von Ungerechtigkeit, Unmenschlichkeit und Zwangsgewalt schmerzhaft und potentiell schädigend ist (s. Abmad et al. 2001; de Zulueta 1993).

Mit der zweiten Studie wurde dieser Ansatz weiterentwickelt, um die offenbar unterschiedliche Neigung von Strafanstalten, Stress unter ihren Gefangenen zu erzeugen, zu untersuchen. Die teilnehmenden Vollzugsanstalten und Stichproben sind in Tab. 4 aufgeführt.

Es zeigte sich, dass die Unterschiede der Mittelwerte bei den Belastungen in allen zwölf Anstalten mit den Suizidraten der jeweiligen Anstalt in einem Dreijahreszeitraum signifikant zusammenhingen (Abb. 1). Die aktuellen Belastungsniveaus deuten in allen

Tabelle 4: Anstalten und Gefangenenstichproben der „Safer Custody Evaluation“ (Liebling et al. 2005)

\begin{tabular}{|l|l|l|}
\hline Anstalt & Gefangene 2002 & Gefangene 2004 \\
\hline Winchester (P) & 100 & 105 \\
\hline Lewes & 104 & 108 \\
\hline Eastwood Park (P) & 103 & 108 \\
\hline Styal & 101 & 117 \\
\hline Leeds (P) & 114 & 113 \\
\hline Liverpool & 118 & 117 \\
\hline Wandsworth (P) & 108 & 109 \\
\hline Manchester & 99 & 107 \\
\hline Feltham (P) & 115 & 95 \\
\hline Glen Parva & 119 & 105 \\
\hline Swansea & 119 & 117 \\
\hline Forest Bank & 101 & 106 \\
\hline Insgesamt & 1.301 & 1.307 \\
\hline
\end{tabular}

$P=$ Anstalten der Pilotstudie

\section{Abbildung 1}

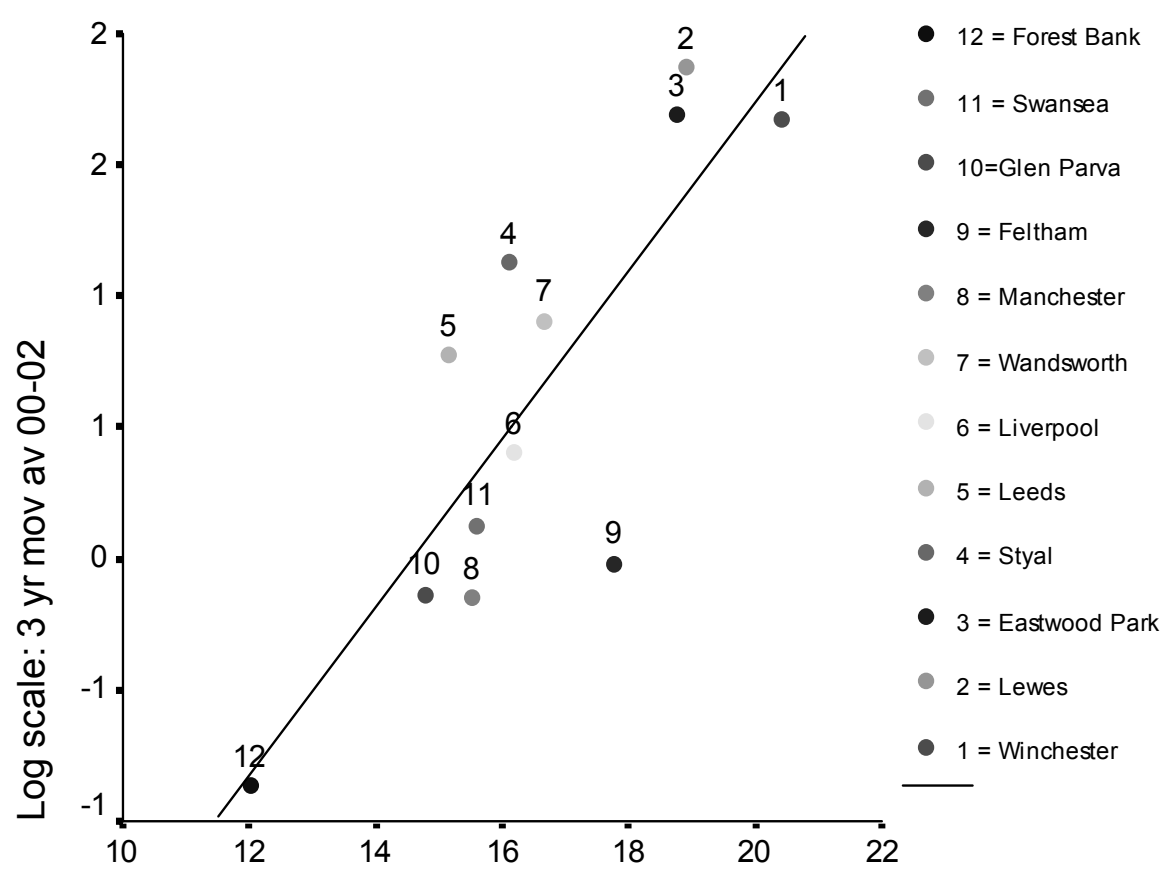

GR new mean GHQ12 score (2002)

Anstalten auf ein mittelfristiges Suizidrisiko hin.

$20 \%$ der Befragten berichteten, „schon einmal in dieser Anstalt an Suizid gedacht zu haben“. Die Anteile schwankten zwischen $9 \%$ in Forest Bank (einem privaten Gefängnis) im Jahr 2002 bis $42 \%$ in Styal (einer kommunalen Frauenstrafvollzugsanstalt) 2004. Im Vergleich acht kommunaler Gefängnisse für männliche Erwachsene für das
Jahr 2002 lagen die Werte zwischen 9\% in Forest Bank und 34\% in Winchester. Die Häufigkeit der Angabe, dass „die Erfahrung des Gefangenseins in dieser Anstalt stressbelastet ist", reichte von 31\% in Forest Bank (2002 und 2004) bis 65\% in Winchester (2004).

Als Messinstrument für Belastung wurde der General Health Questionnaire verwendet, ein Screening-Instrument für schwere 
psychische Störungen wie Angststörung oder Depression. Die Mittelwerte der zufällig ausgewählten Gefangenenstichproben wiesen große Unterschiede auf und reichten im Jahr 2002 von 11,99 in Forest Bank bis 20,16 in Winchester. Ein Wert von 12 oder 13 ist die Grenze, ab der jemand aus der Allgemeinbevölkerung zur genaueren Untersuchung an einen Psychiater verwiesen würde. Nicht zu vergessen ist die Tatsache, dass dies Mittelwerte sind, was auf eine deutlich erhöhte Ausprägung der Belastungen bei manchen Gefangenen hindeutet. Der Anteil der Gefangenen, die der Aussage „zu Beginn meiner Strafe in dieser Anstalt fühlte ich mich gut betreut und versorgt" zustimmten, lag in der Liverpooler Anstalt bei $13 \%$, in Swansea dagegen bei $55 \%$.

Die hohe und unterschiedliche Ausprägung von Belastungen unter den Gefangenen der zwölf Anstalten kann zu einem wesentlichen Teil mit der Bewertung der Gefangenen von Merkmalen des Vollzugs wie Sicherheit, Fairness, Aktivitäten und Respekt erklärt werden (s. Liebling et al. 2005; Liebling 2007). Diese Konzepte standen in einem Zusammenhang, so dass die wahrgenommene Fairness mit dem Niveau gemessenen Vertrauens verknüpft war und Vertrauen wiederum mit dem Wohlbefinden. In der Literatur finden sich Erklärungsansätze für diese Zusammenhänge. Die Teilnahme an Behandlungsprogrammen, andere Maßnahmen zur Persönlichkeitsentwicklung und regelmäßiger Kontakt zur Familie trugen zu erhöhten Werten beim Wohlbefinden bei. Am wichtigsten war jedoch in allen Anstalten die wahrgenommene Sicherheit, die allerdings nicht hauptsächlich mit Risiko und Gefahr zusammenhing, sondern vielmehr mit der Art und Qualität der Beziehungen zwischen Gefangenen und Bediensteten sowie mit Fairness und Vertrauen. Dort, wo die Bediensteten die Gefangenen anständig, respektvoll und fair behandelten, wurde von größerem Vertrauen in die Umgebung, größerer Zuversicht, in allen Belangen auf Bedienstete zugehen zu können und von weniger Frustration berichtet. Auf die Frage, was am meisten zu einem Gefühl von Sicherheit beitrage, antworteten Gefangene mit „Bestätigung “ und „nicht angeschrien zu werden, weißt du? “. Positive Interaktionen und ein Gefühl der Zugänglichkeit führten zu größerem Vertrauen und verbesserten die Wahrscheinlichkeit echter Kommunikation. Gleichgültigkeit oder Feindseligkeit dagegen führten zu Unsicherheitsgefühlen, Hilflosigkeit, Machtlosigkeit, Anspannung und Frustration.
In der Studie, aus der diese Beobachtungen hervorgingen, schwankten die Anteile der Gefangenen, die dem Satz „das Regime in dieser Anstalt ist fair “ zustimmten, zwischen $20 \%$ in Liverpool und $52 \%$ in Swansea. ${ }^{6}$ Als Ungerechtigkeit wurden Machtmissbrauch und Respektlosigkeit erlebt - vertraute und unwillkommene Erfahrungen, die Selbstzweifel und den manchmal heftigen Verlust emotionaler Selbstkontrolle auslösten. Gallo und Ruggiero (1991) sind der Auffassung, dass erheblicher Stress durch das Bemühen ausgelöst werde, diese Gefühle unter Kontrolle zu halten. In der begrenzten Umgebung in der Anstalt war es geringfügig sicherer, seine Frustrationen an sich selber auszulassen als an den Bediensteten oder Mitgefangenen. ${ }^{7}$

Die innere Rechtmäßigkeit oder moralische Leistung hatten also einen Einfluss auf die „Überlebbarkeit“ der Gefangenschaft.

Überdenkt man das Konzept des Wohlbefindens unter Berücksichtigung dieser Ergebnisse, dann gibt es unzählige Analysen über die Zusammenhänge zwischen Rechtschaffenheit oder Anständigkeit und Moral und dem Wohlbefinden des menschlichen Geists angefangen bei Aristoteles' Nikomachischer Ethik (Barnes 1984) bis Alistair McIntyres After Virtue (2000). Sowohl als einzelne als auch als Gruppe leiden wir, wenn Autorität schlecht ausgeübt wird und wir gedeihen und blühen auf, wenn sie umsichtig genutzt wird, wenn Sensibilität und Aufmerksamkeit für andere besteht (Grayling 2003; McIntyre 1999) und wenn sich darin bürgerliche Tugenden widerspiegeln. Eine gute Ausübung der Staatsgewalt hat bessere Folgen (Mulgan 2007). Aus ähnlichen Gründen spielt auch die „gefühlte Qualität der Erfahrung“ (Grayling 2003: 6) innerer Rechtmäßigkeit im Strafvollzug eine Rolle. ${ }^{8}$ Fairness, Respekt und zukunftsorientierte Aktivität tragen erheblich zum Wohlbefinden im Vollzug bei.

Andererseits schreibt Isaiah Berlin so eloquent, dass wir die Nicht-Perfektionierbarkeit des Menschen und seiner Strafanstalten im Sinn behalten und uns damit abfinden sollten (Berlin 1990). ${ }^{9} \mathrm{Zu}$ glauben, das Gefängnis repariere einfach und der Strafvollzugsbedienstete stehe über der Korrumpierbarkeit, ist naiv und gefährlich. Das Gefängnis ist eine „tragische Einrichtung“, im Kern fehlerhaft (Garland 1990). Wir suchen nach Lücken unterschiedlicher Art und Größe und versuchen, sie zu erklären. Wir wissen bereits viel über den Unterschied zwischen Vorzeigebediensteten und solchen, die eher
Schaden anrichten. Nach William Muir (1977) ist ein Strafvollzugsbediensteter wie der beste Polizist gut oder effektiv je nach dem, wie weit er zwei Tugenden entwickelt hat: ein Verständnis für die Natur menschlichen Leidens und moralische Ausgeglichenheit; er hat eher eine tragische als eine ,zynische" Sicht der menschlichen Natur. Darin liegen wichtige moralische Lehren, abgeleitet aus empirischer Forschung.

Die dritte Studie auf unserer kleinen Forschungsreise bestand aus vorläufigen und verblüffenden Untersuchungen der Unterschiede zwischen öffentlichen und privaten Strafvollzugsanstalten in einigen dieser Bereiche des Lebens im Vollzug. Es war z. B. bezeichnend, dass es in den privaten Institutionen im Jahr 2004 keine Suizide gab und dass die Gefangenen in den wenigen privaten Anstalten, die wir über die Jahre in verschiedenen Forschungsprojekten berücksichtigt hatten, ein stärkeres Wohlbefinden berichteten als die Gefangenen aus vergleichbaren öffentlich betriebenen Anstalten. Dies schien mit erkennbaren kulturellen Unterschieden zwischen öffentlichen und privaten Anstalten zusammenzuhängen, u. a. bessere Einstellungen der Bediensteten den Gefangenen gegenüber in einem früheren Stadium der Entwicklung (s. Shefer/Liebling 2008). Gefangene in Doncaster z. B. beschrieben zumindest 2001 einen höchst respektvollen Umgang, ein qualitativ gutes Regime und ein Anstaltsklima, dass ihnen ermöglichte, „sie selbst zu sein“. Dies war nicht unproblematisch und ging mit einer Laxheit bei der Durchsetzung der Anstaltsregeln einher (Liebling, mit Unterstützung von Arnold 2004). Empirische Untersuchungen in Doncaster, Forest Bank und anderen privat betriebenen Anstalten lieferten Belege dafür, dass es möglicherweise wichtige Unterschiede in der sozialen Wirkung und Dynamik privater Strafvollzugsanstalten gibt sowie Abweichungen bei gleichartigen öffentlichen Institutionen. Diese Unterschiede sind messbar und bedeuten, dass Gefangene je nach Standort ganz unterschiedliche Bestrafungsformen und Ausprägungen von Leid erleben. Diese Unterschiede können Folgen für Gefangene haben, unter anderem Suizid, Wut, den Verfall sozialer Beziehungen und gestörte Identitäten.

Als Ergebnis unserer Beratungen in der Forschungsgruppe und unter Berücksichtigung der Arbeit anderer Strafvollzugsforscher unseres Instituts haben wir begonnen, bei der Operationalisierung und dem Versuch der Evaluation des Anstaltsklimas in Be- 
griffen wie „Tiefe“, „Gewicht“ und „Enge“ sowie Sicherheit, Beziehungen, persönliche Entwicklung und Wohlbefinden zu denken. Strafanstalten unterscheiden sich in diesen wichtigen Dimensionen. Da sich unsere Instrumente zur Untersuchung der Einschätzungen und Wahrnehmung der Bediensteten, Lebensqualität und Beziehungen zur Anstaltsleitung verbessern, beginnen wir nun, mögliche Verknüpfungen der Wahrnehmungen und Erfahrungen von Anstaltspersonal und Gefangenen zu erkennen (s. z.B. Gadd et al. submitted).

Die vierte Studie, begonnen Anfang 2007, beinhaltet eine sorgfältig konzipierte Untersuchung, die es uns ermöglicht, diese Überlegungen systematisch weiter zu untersuchen. Sie wird vom Economic and Social Research Council finanziert und läuft unter dem Titel Values, Practices and Outcomes in Public and Private Sector Corrections (Werte, Praxis und Folgen des öffentlichen und privaten Strafvollzugs - d. Übers.). Sie besteht aus drei zusammenhängenden Untersuchungen über die beruflichen Ausrichtungen, Vergangenheit, Praktiken und Prioritäten der Mitglieder von Anstaltsleitungen beider Sektoren, aus einer ethnographischen Studie von zwei öffentlichen und zwei privaten Strafvollzugsanstalten und aus Fallbeispielen zu Maßnahmen zur Modernisierung der Verwaltung (wie z. B. Leistungsüberprüfung oder Eigentümer- bzw. Leitungswechsel). ${ }^{10}$ Dies soll uns ermöglichen, die Auswirkungen neuer und unterschiedlicher Leitungsund Verwaltungsformen auf Verhalten, Einstellungen und Erfahrungen von Bediensteten und Gefangenen zu beschreiben. Wir hoffen, dass die Reichweite unserer Untersuchungen dieses Mal über die Gefängnismauern hinausgeht, damit wir einen Beitrag leisten können zu der von Lanza Kaduce et al. (1999) angestoßenen Debatte über Folgen unterschiedlicher Vollzugsformen und ihre Beziehung, sofern eine besteht, zur inneren moralischen Leistung und Organisation einzelner Strafanstalten. Unsere nach und nach zum Vorschein kommenden Ergebnisse deuten darauf hin, dass der Gebrauch von Autorität durch das Anstaltspersonal eine zentrale Dimension des Lebens im Strafvollzug darstellt. Sie ist wiederum mit Beziehungen verknüpft, und diese beiden wichtigen $\mathrm{Di}$ mensionen können je nach Sektor (also öffentlich oder privat) variieren. Wir hoffen, unsere Ergebnisse dazu demnächst veröffentlichen zu können.

\section{Ausblick}

Derartige Forschungsprojekte sind besonders wichtig in Anbetracht der sich ändernden Größe und Zusammensetzung der Gefangenenpopulation, der Einführung von Wettbewerb und Privatisierung, des Versprechens neue, große Strafvollzugsanstalten zu bauen und des sich ändernden emotionalen und politischen Tons im Bestrafungsdiskurs. Ich habe bereits an anderer Stelle ausgeführt, dass solche Veränderungen der strafrechtlichen Befindlichkeiten das Leben innerhalb der Gefängnismauern maßgeblich formen (Liebling, mit Unterstützung von Arnold 2004). Indem der Gebrauch des Freiheitsentzugs, seine Länge und die politische Reaktionsfreudigkeit auf Ängste der Öffentlichkeit zunehmen, wird das Bedürfnis nach der empirischen Erforschung und theoretischen Betrachtung der allgemeinen psychologischen Auswirkungen des Strafvollzugs immer dringender.

Unser Enthusiasmus, den Gebrauch des Strafvollzugs auszudehnen, hat den Diskurs über seine Wirkungen und Folgen gefährlich eingeengt. Indem der Strafvollzug als Lösung für das Problem der Unsicherheit der Öffentlichkeit angesehen wird, drängt zu leicht in den Hintergrund, dass Strafvollzugsanstalten komplexe, mit Problemen behaftete, oft schädigende Umgebungen sind, deren innere Ordnung unsicher ist. Freiheitsentzug sollte keine unnötigen oder extremen Belastungen zufügen, die über die unvermeidlichen Folgen des Verlustes der Freiheit hinausgehen, ebenso wenig wie er die Fähigkeit eines Straftäters, ein verantwortungsvoller Bürger zu sein, untergraben sollte. Der Freiheitsentzug sollte diese Dinge nicht zufällig oder in extrem unterschiedlichem Ausmaß bewirken. Gefangenschaft und die damit einhergehenden Erfahrungen sollten nicht aus politischen Gründen erschwert oder weniger erträglich gemacht werden. Wir sollten auf der Hut sein beim zunehmenden Gebrauch von Freiheitsentzug überall dort, wo soziale und wirtschaftliche Entbehrungen ungleich verteilt sind. Wir sind heute all dieser und weiterer Verletzungen der Rechtmäßigkeit schuldig, so dass wir die Ungerechtigkeit und das menschliche Leiden verstärken, anstatt sie einzuschränken, wenn wir bestrafen. Es müssen neue Fragen gestellt werden, sowohl über die sich ändernden Erfahrungen einiger neuer Gefangenengruppen - aufgrund eines Bewährungswiderrufs Inhaftierte und Gefangene mit zeitlich unbestimmten Sanktionen, oder sichtbare Minderheiten und fremde Nationalitäten -, als auch über die offensichtlich großen Unterschiede zwischen Strafvollzugsanstalten. Die Auseinandersetzung mit den Auswirkungen des Strafvollzugs ist ein vernachlässigtes und wenig beachtetes Thema, das in der Forschung Priorität haben sollte. Unserer Liste der Tugenden sollten noch Folgende hinzugefügt werden: „eingehende, dauerhafte und eifrige Beobachtung“ (s. Grayling 2003: 7), denn das ist unsere Aufgabe.

Alison Liebling ist Professorin für Kriminologie und Strafrechtspflege und Direktorin des Zentrums für Strafvollzugsforschung an der Universität Cambridge in England. Sie dankt Frieder Dünkel für die Einladung, diesen Aufsatz zu schreiben, Sarah Tait, Linda Durie, Gerry Rose und Annick Stiles für die Mitarbeit im Safer Custody Project sowie Dirk van Zyl Smit und Nicky Padfield für hilfreiche Korrespondenz.

Übersetzung von Philip Horsfield und Kirstin Drenkhahn.

\section{Literatur}

Ahmed, E., Harris, N., Braithwaite, J., Braithwaite, V. (2001): Shame Management Through Reintegration. Cambridge: Cambridge University Press.

Aristotle's 'Ethics'. In: Barnes, J. (ed.) (1984): The Complete Works of Aristotle: The Revised Oxford Translation New Jersey: Princeton University Press.

Arnold, H., Liebling, A., Tait, S. (2007): Prison Officers and Prison Culture. In: Jewkes, Y. (ed.): The Prisons Handbook. Cullompton: Willan.

Berlin, I. (1990): The Crooked Timber of Humanity. Edited by H. Hardy. New Jersey: Princeton University Press.

Boyle, J. (1984/1985): The Pain of Confinement: Prison Diaries. London: Pan.

Churchill, W., speech to House of Commons 20 July 1910, Reported in the New York Times August 71910 - Reforming the Prison System of Great Britain.

Cohen, S., Taylor, L. (1972): Psychological Survival: The Experience of Long-Term Imprisonment. Harmondsworth: Penguin.

de Zulueta, F. (1993): From Pain to Violence: The Traumatic Roots of Destructiveness. London: Whurr.

Deflau, M. (2001): Review of 'Les Prisons de la Misère by Loïc Wacquant'. Punishment and Society, 3(2), pp. 307-9.

Gadd, V., McLean, C., Liebling, A., Shefer, G., Tait, S. (submitted): Measuring staff quality of life: Implications for research on prison culture (under review).

Gallo, E., Ruggiero, V. (1991): The "Immaterial" Prison: Custody as a Factory for the Manufacture of Handicaps. International Journal of the Sociology of Law, 19 (3), pp. 273-291.

Garland, D. (1990): Punishment and Modern Society. Oxford: Clarendon Press.

Grayling, A. C. (2003): What is Good? London: Phoenix.

Haney, C. (1997): Psychology and the Limits to Prison Pain: Confronting the Coming Crisis in the Eighth Amendment Law. Psychology, Public Policy and Law, 3 (4), pp. 499-588. 
Horn, M. F. (2004): The Implications of Abu Ghraib for the American Prison System. Remarks delivered at the Vera Institute of Justice June 22, 2004.

Johnson, R., Toch, H. (1982): The Pains of Imprisonment. London; Beverley Hills CA.: Sage.

Lanza-Kaduce, L. Parker, K., Thomas, C. (1999): A Comparative Recidivism Analysis of Releases from Private and Public Prisons. Crime and Delinquency, 45: 28-47.

Lazarus, L. (2004): Contrasting Prisoners' Rights: A Comparative Examination of England and Germany, Oxford: Oxford University Press.

Liebling, A. (2007): Why Prison Staff Culture Matters. In: J. M. Byrne, D. Hummer and F. S. Taxman (eds.): The Culture of Prison Violence. New York: Pearson, pp. 105-22.

Liebling, A. assisted by Arnold, H. (2004): Prisons and their Moral Performance: A Study of Values, Quality and Prison Life. Oxford: Clarendon Press.

Liebling, A., Durie, L., Stiles, A., Tait, S. (2005): Revisiting prison suicide: the role of fairness and distress. In A. Liebling and S. Maruna (eds): The Effects of Imprisonment. Cullompton: Willan, pp. 209-31

Lippke, R. L. (2007): Rethinking Imprisonment. Oxford: University Press.

Loader, I. (2008): Straw's embrace of penal excess ignores the public will. Guardian.co.uk - Tuesday October 28, 2008.

MacIntyre, A. (1981/2000): After Virtue: A Study in Moral Theory. $10^{\text {th }}$ edn. Duckworth, London.

MacIntyre, A. (1999): Dependent Rational Animals: Why Human Beings Need the Virtues. Duckworth, London.

Muir, W. K. (1977): Police: Streetcorner Politicians. University of Chicago Press, Chicago.

Mulgan, G. (2007): Good and Bad Power: The Ideals and Betrayals of Government. London: Penguin

Ruck, S. K. (ed.) (1951): Paterson on Prisons: The Collected Papers of Sir Alexander Paterson. London: Frederick Muller

Shefer, G., Liebling, A. (2008): Prison privatization: In search of a business-like atmosphere? Criminology and Criminal Justice 8(3): 26178.

Smith, A. (1759; 2006 ed.): The Theory of Moral Sentiments. Dover Philosophical Classics.

Sparks, R. (1994): Can Prisons Be Legitimate?. In R. King and M. McGuire (eds): Prisons in Context, Clarendon Press, Oxford.

Straw, J. (2008): Speech on 'Punishment and Reform' to the Royal Society of Arts 27 October 2008, Ministry of Justice.

Sykes, G. (1958): The Society of Captives. Princeton University Press, Princeton, N.J.

Tait, S. (2008): Care and the prison officer: beyond 'turnkeys' and 'carebears'. Prison Service Journal 180: 3-11.

van Zyl Smit, D., Snacken, S. (2009): Principles of European Prison Law and Policy: Penology and Human Rights. Oxford: Oxford University Press.

von Hirsch, A. (1976): Doing Justice: The Choice of Punishments: Report of the Committee for the Study of Incarceration. New York: Hill and Wang.

von Hirsch, A., Ashworth, A. (2005): Proportionate Sentencing: Exploring the Principles. Oxford: Oxford University Press.

Wacquant, L. (1999) : Les Prisons de la Misère. Paris: Editions Raisons d'Agir

Walker, N. (1987): The Unwanted Effects of Long-Term Imprisonment. In A.E. Bottoms and R. Light (eds.): Problems of Long-Term Imprisonment. Gower, Aldershot.

Zimbardo, P. (2007): The Lucifer Effect: How Good People Turn Evil. London: Random House Group; Rider and Co.

\section{Zitierte Rechtsprechung}

Wilberforce, Lord Raymond v Honey [1982] 2 WLR 465

R v Secretary of State for the Home Department Ex p. Sims [2000] 2 A.C. 115 [1999] 3 W.L.R. $328=$ House of Lords

$\mathrm{R}$ (on the application of Nilsen) v Full Sutton Prison Governor [2004] EWCA Civ 1540. [2005] 1 W.L.R. 1028 = Court of Appeal.

Dickson v United Kingdom [2007] Application no. $44362 / 04=$ Council of Europe

\section{Fußnoten:}

1 In Deutschland hat das BVerfG die Resozialisierung als Hauptziel des Strafvollzugs identifiziert. Dies wird als ein der Menschenwürd innewohnendes Element sowie als wesentlicher Wert in einem Sozialstaat betrachtet (van Zyl Smit/Snacken 2009). Das Resozialisierungsprinzip findet seinen legislativen Ausdruck im Strafvollzugsgesetz von 1976. Liora Lazarus bemerkt dazu, dass die ausdrückliche Anerkennung der Resozialisierung als Primärziel des Strafvollzugs durch das BVerfG (unabhängig von anderen Strafzwecken) einen besseren Schutz von Gefangenenrechten ermöglicht als den, den die englischen Gerichte oder der Europäische Gerichtshof für Menschenrechte gewährleisten können (Lazarus 2004; s, auch van Zyl Smit/Snacken 2009).

2 S. auch R v Secretary of State for the Home Department ex p. Sims [2000]; R (on the application of Nilsen) v Full Sutton Prison Governor [2004]; Dickson v The United Kingdom [2007]. Dabei wird die Unterscheidung zwischen ,persönlicher" Freiheit und Menschenrechten ak zeptiert (van Zyl Smit/Snacken 2009).

3 ,There is a treasure if you can only find it in the heart of every man", so Churchill in seiner Rede im Britischen Unterhaus am 20.7.1910. Seine Rede wurde beeinflusst von John Galsworthys Theaterstück Justice, das die schrecklichen Folgen von Isolationshaft aufzeigt (New York Times, 7.8.1910: Reforming the Prison System of Great Britain). Churchills Grundannahmen waren, dass Gefängnisse nicht herabwürdigen, sondern bessern sollen und dass Sanktionen nicht nur vergeltend sein sollten (insbesondere bei jungen Menschen). Er schlug u. a. vor, vierteljährlich Konzerte oder Vorträge innerhalb der Anstalt abzuhalten. Zudem sei Musik ein Trost, der keinem staatlichen Strafzweck zuwider laufen könne. Im Gegensatz dazu erklärte Jack Straw, dass er einen Comedy-Kurs in der Strafanstalt Whitemoor für ,absolut inakzeptabel" halte (Europe News, 22.11.2008).

4 S. z. B. Adam Smith (1759) und Alistair McIntyre (2000). In seiner Rede beharrte Jack Straw (2008) darauf, dass der Gebrauch einer "Sprache der Bestrafung“ nicht mit einem „unmenschlichen Umgang mit Straftätern “ gleich zusetzen sei. Aber, wie Martin Horn festhält, „die Sprache/Ideologie der Bestrafung ist ein Beschleuniger der Brutalität“, und „die Füh rungsebene eine Kultur erzeugt, die Gefangene als Bürger und Menschen mit Rechten definiert. Wenn sie es nicht macht, macht es niemand" (Horn 2004, S. 2). Straw (2008) äußerte auch sein „Entsetzen“ über eine Halloween-Feier in einem Frauengefängnis. Forscher sollten sich nicht davor fürchten, ebenfalls eine moralische Sprache zu verwenden. Die Befunde deuten darauf hin, dass das moralische Klima durchaus bedeutsam ist (s. u.).

5 Insgesamt betrachtet konnten weniger als die Hälfte der befragten Häftlinge „in dieser Anstalt sie selbst sein". Haney (1997: 537) meint, dass Gefangene, die sich sowohl auf der emotionalen als auch auf der Verhaltensebene bemühen, eine undurchlässige und das wahre Gesicht verschleiernde Anstaltsmaske zu erzeugen,
Entfremdung, emotionale Abstumpfung und Distanz und den Rückzug aus sozialen Interaktionen riskieren.

$6 \mathrm{Im}$ „besten Gefängnis“ stimmte nur die Hälfte der Gefangenen der Aussage zu, dass das Regime dort fair sei. Ein sehr schlechtes Gefängnis hat seine Bewertung von 2002 bis 2004 um $16 \%$ verbessert, von 31 auf $47 \%$. Diese Anstalt wurde durch die Gefangenen auch als deutlich fürsorglicher beschrieben, und die Häufigkeit ernsthafter Selbstverletzungsversuche und von Suiziden nahm ab.

7 Scheinbar kleine Ereignisse wie z. B. das Telefonieren konnten unzählige Hindernisse, Ignoriertwerden und Ablehnung mit sich bringen, bevor sie durch die Mitwirkungen eines freundlicheren Bediensteten ermöglicht wurden. Diese Hindernisse traten in manchen Gefängnissen mehr auf als in anderen. Anstalten danach zu differenzieren erwies sich als fruchtbar be unseren Versuchen, die Kultur des jeweiligen Anstaltspersonals $\mathrm{zu}$ beschreiben und einzuschätzen (s. Liebling 2007; Arnold/Liebling/ Tait 2007; Tait 2008). Die Erfahrung von Ungerechtigkeit griff auch auf Personen außerhalb der Anstalt - Familien - über, die oft z. B. nich wussten, dass ein Telefonat nicht möglich war, weil der abendliche Aufschluss gestrichen wa oder dass ein Gefangener oder eine Gefangene in der Nacht zuvor in eine andere Anstal verlegt worden war und daher nicht zu einem Besuchstermin erscheinen konnte.

8 S. Liebling mit Unterstützung von Arnold 2004 Die Frage der äußeren Rechtmäßigkeit und wie oder in welchem Ausmaß sie von Gefangenen erlebt wird, ist ein wichtiges und verwandte Thema, das jedoch den Rahmen dieses Berichts sprengen würde (s. Sparks 1994).

9 „No more rigorous moralist than Immanuel Kant has ever lived, but even he said, in a moment of illumination, 'out of the crooked timber of humanity no straight thing was ever made'. To force people into the neat uniforms demanded by dogmatically believed-in schemes is almost always the road to inhumanity. " $(\mathrm{Ber}$ lin 1990: 19, der Collingwoods Kant-Übersetzung zitiert).

10 Der Begriff „Modernisierung“ bezieht sich au eine Reihe von Änderungen in der Verwaltung, die seit den späten 1980er Jahren im Strafvollzug mit dem Ziel stärkerer Professionalisierung in einigen Bereichen und größerer Verantwortlichkeit für Finanzen und Führung stattfanden. 